\title{
Correlation analysis of Ki67 and CK7 expression with clinical characteristics and prognosis of postoperative cervical adenocarcinoma patients
}

\author{
Yuzhong Wu, Mengmeng Lv, Tianye Qian, Yang Shen \\ Department of Gynecologic Oncology, Jiangsu Cancer Hospital and Jiangsu Institute of Cancer Research and The Affiliated Cancer Hospital of \\ Nanjing Medical University, Nanjing, China \\ Contributions: (I) Conception and design: Y Wu; (II) Administrative support: None; (III) Provision of study materials or patients: T Qian; (IV) \\ Collection and assembly of data: Y Shen; (V) Data analysis and interpretation: M Lv; (VI) Manuscript writing: All authors; (VII) Final approval of \\ manuscript: All authors. \\ Correspondence to: Yang Shen. Department of Gynecologic Oncology, Jiangsu Cancer Hospital and Jiangsu Institute of Cancer Research and The \\ Affiliated Cancer Hospital of Nanjing Medical University, No. 42 Baiziting Street, Nanjing 210009, China. Email: sykaxh@126.com.
}

\begin{abstract}
Background: The purpose of this study is to investigate the correlation between cytokeratin 7 (CK7) and marker of proliferation Ki67 protein expression and clinical characteristics and prognosis of patients with cervical adenocarcinoma after surgery.

Methods: A total of 126 patients with cervical adenocarcinoma treated by surgery in our hospital from June 2011 to September 2015 were enrolled in this study. Immunohistochemistry (IHC) was used to detect the expression of CK7 and Ki67 in 126 cases of cervical adenocarcinoma tissues. The chi-square $\left(\chi^{2}\right)$ test was used to compare the relationship between the positive expression rate of CK7 or Ki67 and clinicopathological features. Kaplan-Meier method was used to analyze the survival of different protein expression groups and Cox proportional hazards regression model was used to analyze the risk factors affecting the prognosis.

Results: The positive rate of CK7 was correlated with muscle invasion, vascular invasion, differentiation, and lymph node metastasis (all $\mathrm{P}<0.05$ ). The positive expression rate of $\mathrm{Ki67}$ was related to the degree of myometrial invasion and International Federation of Gynecology and Obstetrics (FIGO) stage (both $\mathrm{P}<0.05)$. Both CK7 and Ki67 may be independent risk factors for the prognosis of patients with cervical adenocarcinoma after surgery (both $\mathrm{P}<0.05$ ), and their high expression heralds worse prognosis.

Conclusions: The CK7 and Ki67 proteins may be key regulatory factors in the development of cervical adenocarcinoma after surgery, and their overexpression may lead to worse prognosis. Both CK7 and Ki67 may provide new markers for prognosis evaluation of cervical adenocarcinoma.
\end{abstract}

Keywords: Cervical adenocarcinoma; Ki67; cytokeratin 7 (CK7); prognosis

Submitted Jun 25, 2021. Accepted for publication Aug 17, 2021.

doi: 10.21037/apm-21-1974

View this article at: https://dx.doi.org/10.21037/apm-21-1974

\section{Introduction}

Cervical cancer is one of the most common gynecological malignancies, ranking the fourth in female malignancies, and is more common in developing countries (1). According to the data in 2018 , there were 569,000 new cases of cervical cancer and 311,000 cervical cancer deaths in the world, of which more than $85 \%$ were in developing countries (2).
As the most populous developing country in the world, there are 130,000 new cases of cervical cancer every year in China (3). Cervical adenocarcinoma is the main pathological type of invasive cervical cancer after cervical squamous cell carcinoma. A worldwide epidemiological survey found that the incidence rate of cervical adenocarcinoma increased annually, and its proportion in cervical cancer 
patients increased from 5\% to 20-25\% (4). Multiple studies have shown that adenocarcinoma accounts for a higher proportion of cervical cancer than other pathological types in young patients (5-7). About $10 \%$ of cervical adenocarcinoma is not accompanied by human papilloma virus (HPV) infection, and cervical adenocarcinoma in situ and cervical adenocarcinoma lesions mostly occur in the cervical canal and are segmental and focal. Therefore, cytological specimens are not easy to collect, the pathological diagnosis of adenocarcinoma is more difficult than that of squamous cells, and most patients have already progressed to an advanced stage when diagnosed $(8,9)$. Compared with cervical squamous cell carcinoma, cervical adenocarcinoma generally has worse prognosis, higher recurrence (10), and higher invasion and migration ability, which is a serious threat to women's life and health (11). Therefore, it is of great significance to study the prognostic factors and mechanism of cervical adenocarcinoma. The proliferation, differentiation, and metastasis of cancer cells are a complex reaction process of multi-gene, multistage, and multi-step interaction (12). Cytokeratin 7 (CK7) is a low molecular cytokeratin that is located on or near the surface of the body in the upper cortex. It is expressed differently in different stages of cell differentiation and in different cell types, and is usually regarded as the basic molecular marker protein of epithelial differentiation. Therefore, it can express or partially express the expression pattern of cytokeratin molecules in the cells from which it originated to study the origin and metastasis of tumors. CK7 is mainly expressed in the cytoplasm of cells and is a marker of adenoid differentiation. It mainly exists in glandular epithelium and transitional epithelium, but not in squamous epithelium. It is often used as a marker of cervical adenocarcinoma, ovarian cancer, renal cell carcinoma, or transitional cell carcinoma (13). A large sample study found that the expression rate of CK7 in adenocarcinoma was about $97.3 \%$ (14). Ki67 is an ideal molecular marker to express the proliferative activity of tumor cells. As a nuclear antigen associated with proliferation cells, Ki67 is expressed in ovarian, breast, lung, esophageal cancer and other tumor cells, and the level of its expression is closely related to the degree of malignancy of tumors. The higher the expression level of Ki67, the more likely the early invasion and metastasis of tumor may occur (15).

Therefore, this study recruited patients with postoperative cervical adenocarcinoma in our hospital as the participants to explore the correlation between CK7/Ki67 and different clinicopathological characteristics/prognosis of patients with postoperative cervical adenocarcinoma, so as to provide new markers for the prognosis evaluation of cervical adenocarcinoma. We present the following article in accordance with the REMARK reporting checklist (available at https://dx.doi.org/10.21037/apm-21-1974).

\section{Methods}

\section{Participants}

A total of 126 patients with cervical adenocarcinoma who underwent surgical treatment in our hospital from June 2011 to September 2015 were enrolled. All participants were diagnosed with cervical adenocarcinoma by computed tomography (CT), magnetic resonance imaging (MRI), color Doppler ultrasound, and histopathological biopsy according to the relevant diagnostic criteria of "Guidelines for the diagnosis and treatment of cervical cancer (fourth edition)" (16). The inclusion criteria were as follows: surgical method was radical hysterectomy + bilateral ovariosalpingectomy + radical pelvic lymphadenectomy + abdominal para-aortic lymphadenectomy; no previous history of gynecologic malignant tumor surgery; no preoperative radiotherapy or chemotherapy; follow-up records of more than 5 years were available. All procedures performed in this study involving human participants were in accordance with the Declaration of Helsinki (as revised in 2013). The study was approved by Jiangsu Cancer Hospital (No.: 20110584). All participants and their families were aware of the study, and all participants provided written informed consent before commencement of the study.

\section{Data collection}

\section{Clinicopathological data collection}

The age, tumor diameter $(\leq 4 \mathrm{~cm} />4 \mathrm{~cm})$, interstitial invasion $(<1 / 2$ muscularis $/ \geq 1 / 2$ muscularis), vascular invasion $(\mathrm{Y} / \mathrm{N})$, degree of differentiation (low/moderate/ high), International Federation of Gynecology and Obstetrics (FIGO) stage (stage Ia2-IIa2), and lymph node metastasis $(\mathrm{Y} / \mathrm{N})$ of patients with cervical adenocarcinoma after surgery were collected through the hospital medical record system. All cases were followed up completely, and the main follow-up methods were telephone combined with patients' reexamination in hospital. The time of operation was taken as the start time of survival, and death was taken as the end event. The time interval between the end event time and the start time was defined as the total survival 
time of patients. All participants were followed up until September 2020, and the follow-up rate was $100 \%$.

A total of 126 patients with cervical adenocarcinoma were included in this study. The age of onset was 35-58 years old, with an average age of $46.31 \pm 7.82$ years old, including 58 cases $<46$ years old and 68 cases $\geq 46$ years old. There were 89 cases with tumor diameter $\leq 4 \mathrm{~cm}$ and 37 cases with tumor diameter $>4 \mathrm{~cm} ; 87$ cases infiltrated less than $1 / 2$ mesenchyme and 39 cases infiltrated more than $1 / 2$ mesenchyme; 36 cases of vascular invasion and 90 cases without vascular invasion; 28 cases with high differentiation and 98 cases with moderate and low differentiation. A total of 96 cases were in stage I and 30 cases in stage II (20 cases in $\mathrm{Ia} 2,56$ cases in stage $\mathrm{Ib} 1,20$ cases in stage $\mathrm{Ib} 2,21$ cases in stage IIa1, and 9 cases in stage IIa2) in FIGO stage; 33 cases with lymph node metastasis, and 93 cases without lymph node metastasis.

\section{Light microscope observation}

All samples were fixed with $4 \%$ neutral formaldehyde, dehydrated, paraffin embedded, sliced into $4 \mu \mathrm{m}$ thick sections, stained with hematoxylin and eosin (HE) and observed under a light microscope. Differentiation degree of common adenocarcinoma was classified as follows: highly differentiated adenocarcinoma was mainly composed of glandular duct structure, with solid area $<5 \%$; low differentiated adenocarcinoma, solid region $>50 \%$; and medium differentiated adenocarcinoma was between the others.

\section{Expression of CK7 and Ki67 detected by immunohistochemistry (IHC)}

The cervical adenocarcinoma tissues of all participants were extracted, and the expression of CK7 and Ki67 was detected by IHC. The staining results were scored separately by two experienced clinicians according to the same scoring criteria without knowledge of the section data. The double scoring method was used to judge the results. A total of 10 clear visual fields were selected from each slice to count the number of positive staining cells. The staining intensity was calculated according to the staining characteristics of most cells (the staining depth was compared with the background staining situation): no staining was 0 , light yellow was 1 , brown-yellow was 2 , and brown was 3 . According to the ratio of the number of positive cells [i.e., for each case, the number of positive cells of 5 high power microscopic fields (100x) were randomly observed], the number of positive cells less than $25 \%$ was recorded as $0 ; 15-50 \%$ was scored as 1 point; $50-75 \%$ was scored as 2 points; and $\geq 75 \%$ was recorded as 3 points. The integral product of the two groups was calculated, and the integral $\leq 3$ was taken as negative, and $>3$ as positive.

\section{Statistical analysis}

The software SPSS 26.0 (IBM Corp., Chicago, IL, USA) was used to analyze the data. The counting data were expressed by percentage (\%), and the differences were compared by chi-square $\left(\chi^{2}\right)$ test. The $\chi^{2}$ test was also used to compare the relationship between the positive expression rates of CK7 and/or Ki67 and different clinicopathological features. Kaplan-Meier method was used to analyze the survival of the two groups with different protein expression, and log-rank method was used to test the survival difference between the two groups. Cox proportional hazards regression model was used to analyze the risk factors influencing the prognosis of patients with cervical adenocarcinoma after surgery. A $\mathrm{P}$ value $<0.05$ was deemed statistically significant.

\section{Results}

\section{Microscopic examination results}

The results of microscopic examination showed that all kinds of abnormal cervical glands were more abundant than normal or benign cervical glands infiltrating in the cervical stroma. According to the World Health Organization (WHO, 2014) diagnostic criteria for pathological and genetic classification of female reproductive system tumors, the tumors were classified histologically. In this study, there were 88 cases $(69.84 \%)$ of common adenocarcinoma, 9 cases (7.14\%) of endometrioid adenocarcinoma, 9 cases $(7.14 \%)$ of intestinal mucinous adenocarcinoma, 7 cases $(5.56 \%)$ of serous adenocarcinoma, 6 cases $(4.76 \%)$ of adenocarcinoma with neuroendocrine carcinoma, and 7 cases $(5.56 \%)$ of other types of adenocarcinomas detected by microscopic examination (Figure 1).

\section{IHC staining results}

The results of IHC staining showed that CK7 protein was mainly cytoplasmic staining, while Ki67 protein was nuclear staining. The positive rates of $\mathrm{CK} 7$ and Ki67 protein in neoplastic foci were $74.60 \%$ and $69.84 \%$, respectively (Figure 2). 

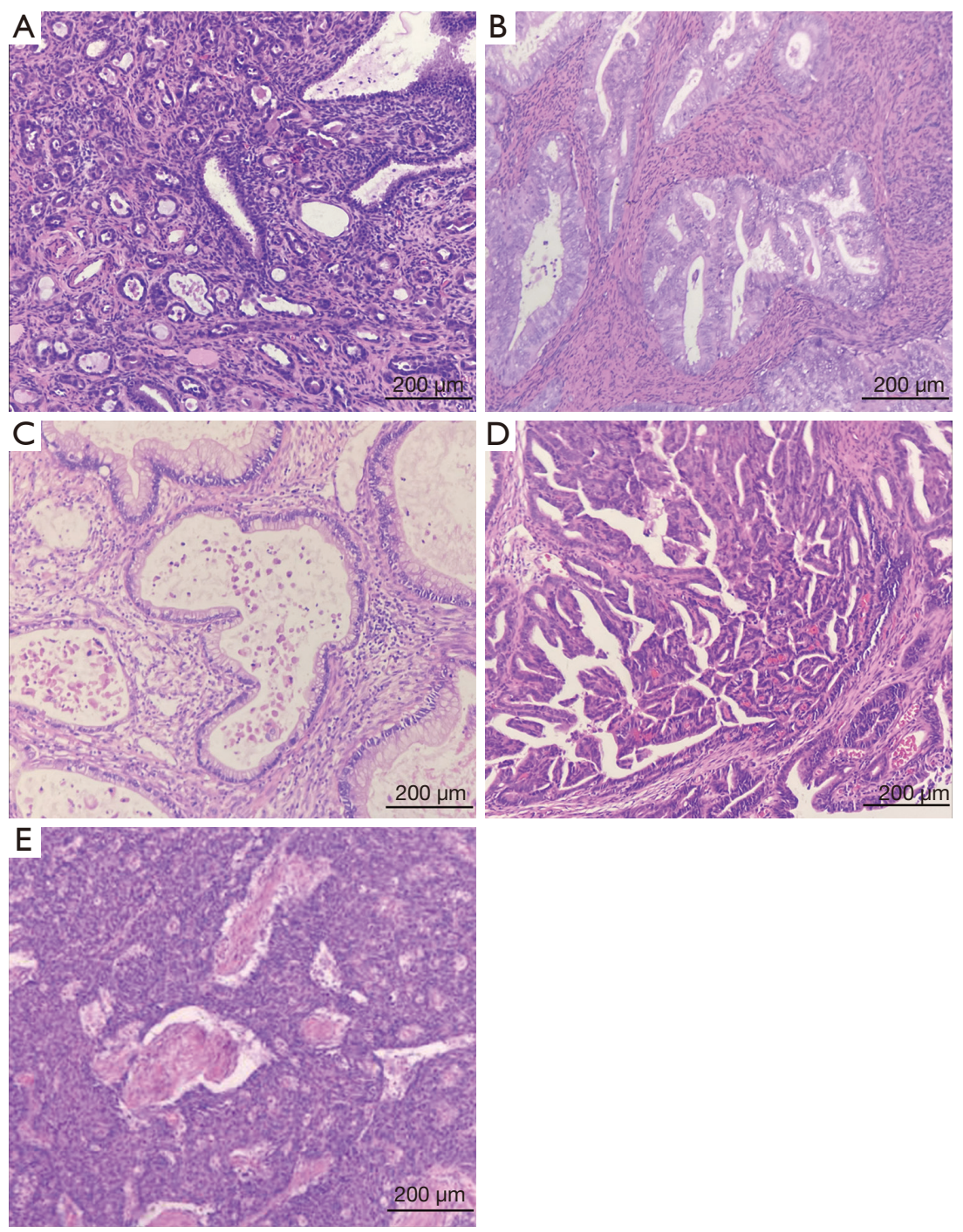

Figure 1 The results of microscopic examination of cervical adenocarcinoma (crystal violet staining, 10×10). (A) Common adenocarcinoma; (B) endometrioid adenocarcinoma; (C) intestinal mucinous adenocarcinoma; (D) serous adenocarcinoma; (E) adenocarcinoma with neuroendocrine carcinoma.

\section{Relationship between CK7, Ki67 positive expression and clinicopathological features of postoperative cervical adenocarcinoma}

There was no significant difference in the positive expression rate of CK7 among different ages, tumor diameter, and FIGO stage of cervical adenocarcinoma patients (all $\mathrm{P}>0.05$ ), but the positive expression rate was correlated with interstitial invasion, vascular invasion, degree of differentiation, and lymph node metastasis (all $\mathrm{P}<0.05)$. The CK7 positive expression rate in participants with $\geq 1 / 2$ myometrial invasion was higher than that in those with $<1 / 2$ myometrial invasion $\left(\chi^{2}=9.34, \mathrm{P}=0.002\right)$; Compared with patients without vascular invasion, CK7 positive rate was higher in participants with vascular invasion $\left(\chi^{2}=5.43, \mathrm{P}=0.020\right)$, and CK7 positive rate in participants with low differentiation was higher than that in those with high differentiation $\left(\chi^{2}=4.10, \mathrm{P}=0.043\right)$. Compared with participants without lymph node metastasis, CK7 positive expression rate in those with lymph node metastasis was higher $\left(\chi^{2}=6.27, \mathrm{P}=0.012\right)$ (Table 1$)$.

There was no significant difference in positive expression rate of Ki67 among different age, tumor diameter, vascular 

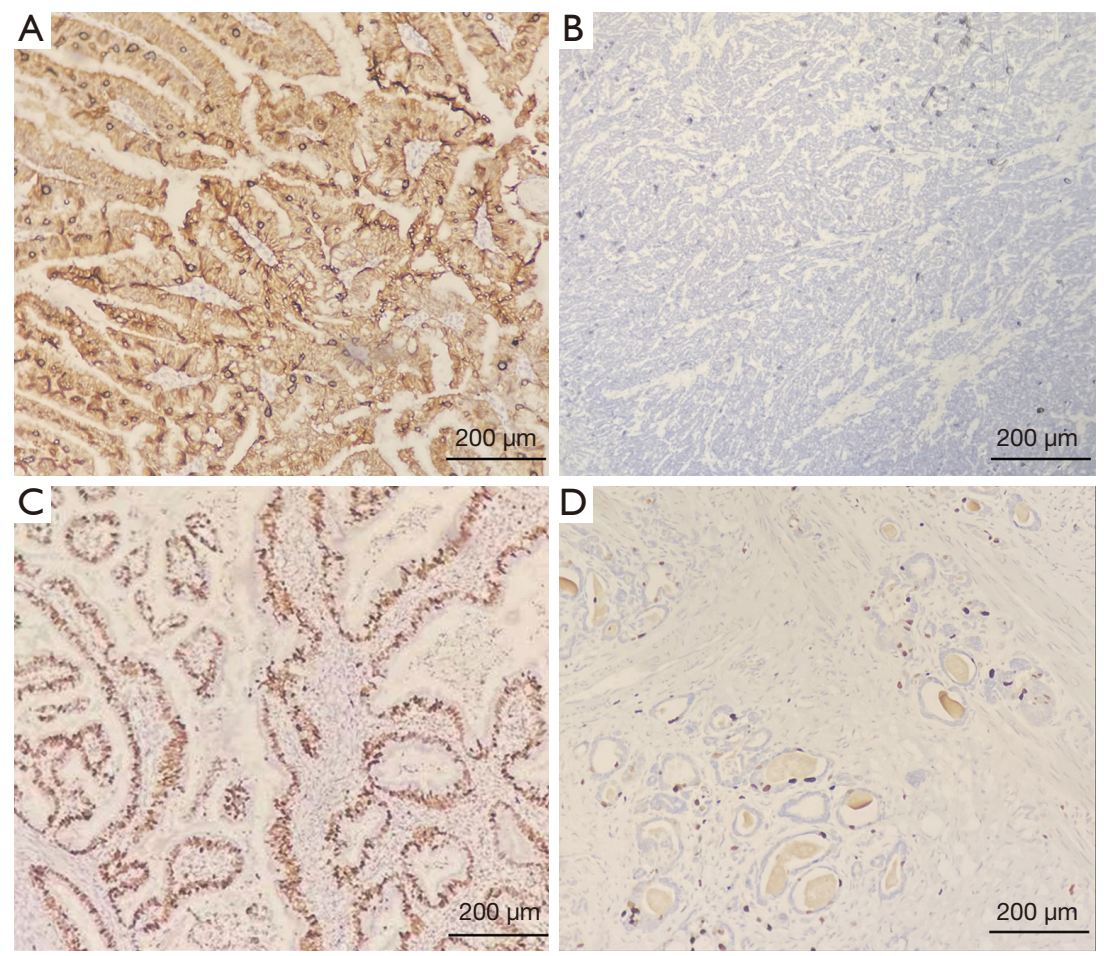

Figure 2 IHC staining of CK7 and Ki67 in cervical adenocarcinoma tissues. (A) IHC staining (10×10) of CK7 protein in cervical adenocarcinoma tissue shows positive; (B) IHC staining $(10 \times 10)$ of CK7 protein in cervical adenocarcinoma tissue shows negative; (C) IHC staining $(10 \times 10)$ of Ki67 protein in cervical adenocarcinoma tissue shows positive; (D) IHC staining $(10 \times 10)$ of Ki67 protein in cervical adenocarcinoma tissue shows negative. IHC, immunohistochemical; CK7, cytokeratin 7.

invasion, differentiation, and lymph node metastasis (all $\mathrm{P}>0.05$ ); Compared with participants with $<1 / 2$ myometrial invasion, those with $\geq 1 / 2$ myometrial invasion had higher positive expression rate of $\mathrm{Ki} 67$, and the difference was statistically significant $\left(\chi^{2}=10.62, \mathrm{P}=0.001\right)$; The positive expression rate of Ki67 in participants with FIGO stage II was higher than that in those with FIGO stage $\mathrm{I}\left(\chi^{2}=5.29\right.$, $\mathrm{P}=0.021$ ) (Table 1).

\section{Cox regression analysis on prognosis of postoperative cervical adenocarcinoma}

Cox regression was used to analyze the factors influencing the prognosis of cervical adenocarcinoma patients after operation. The results of univariate and multivariate analysis both showed that vascular invasion, CK7, and Ki67 may be independent risk factors for prognosis of cervical adenocarcinoma patients after operation (all $\mathrm{P}<0.05)$. Multivariate analysis showed that the prognosis of participants with vascular invasion was worse than that of those without vascular invasion [hazard ratio $(\mathrm{HR})=1.43$, $95 \%$ confidence interval $(\mathrm{CI}): 1.15$ to $2.67, \mathrm{P}<0.001]$. This study further found that patients with positive CK7 and Ki67 expression had a worse prognosis than those with negative $\mathrm{CK} 7$ and Ki67 expression (CK7: HR =2.26, 95\% CI: 1.60 to $3.15, \mathrm{P}<0.001$; Ki67: $\mathrm{HR}=1.63,95 \% \mathrm{CI}$ : 1.39 to $2.70, \mathrm{P}<0.001$ ) (Table 2).

\section{Correlation between vascular invasion and overall survival (OS) rate of postoperative cervical adenocarcinoma patients}

Results of Kaplan-Meier survival analysis showed that the median survival time of participants with vascular invasion was 35.86 (33.15-38.57) months, lower than the 44.36 (41.96-46.77) months of patients without vascular invasion. The OS was better in participants without vascular invasions than in those with vascular invasions $(\mathrm{P}<0.001)$ (Figure 3). 
Table 1 Difference of CK7 and Ki67 positive expression in cervical adenocarcinoma with different clinicopathological features

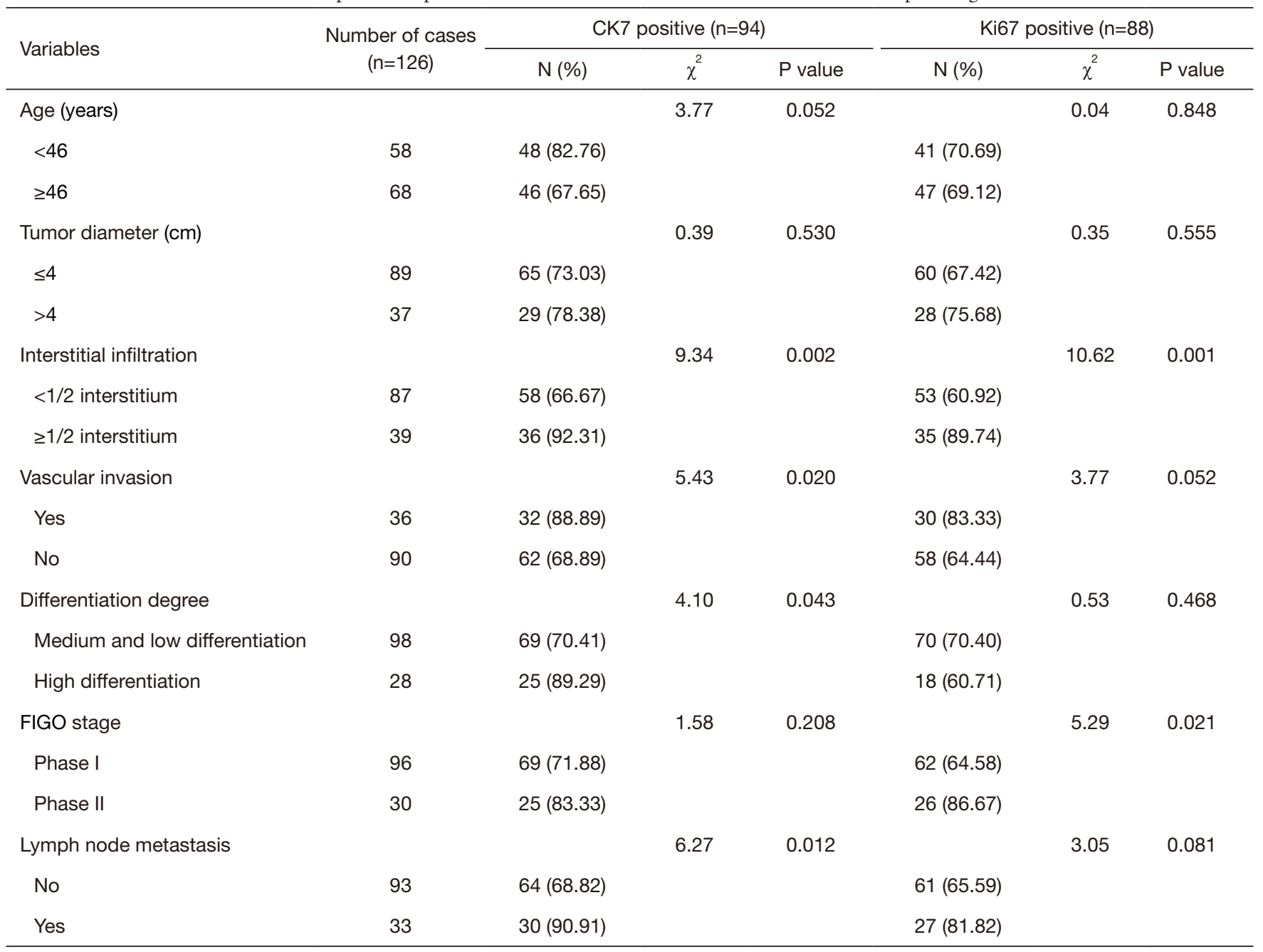

CK7, cytokeratin 7; FIGO, International Federation of Gynecology and Obstetrics.

Table 2 Cox regression analysis on prognosis of postoperative cervical adenocarcinoma

\begin{tabular}{|c|c|c|c|c|c|c|}
\hline Variables & \multicolumn{3}{|c|}{ Univariate } & \multicolumn{3}{|c|}{ Multivariate } \\
\hline Vascular invasion & 1.90 & $1.29-2.78$ & $<0.001$ & 1.43 & $1.15-2.67$ & $<0.001$ \\
\hline CK7 & 2.14 & $1.38-3.31$ & $<0.001$ & 2.26 & $1.60-3.15$ & $<0.001$ \\
\hline Ki67 & 1.67 & $1.11-2.51$ & $<0.001$ & 1.63 & $1.39-2.70$ & $<0.001$ \\
\hline
\end{tabular}

* indicates that confounders (age) have been corrected. HR, hazard ratio; Cl, confidence interval; CK7, cytokeratin 7.

\section{Relationship between positive expression of CK7 and Ki67 and survival rate of cervical cancer patients after operation}

Results of Kaplan-Meier survival analysis showed that the median survival time of patients with negative CK7 expression was 46.27 (43.56-48.98) months, which was higher than the 36.78 (34.48-39.08) months of those with positive CK7 expression. Patients with negative CK7 expression had better OS than those with positive CK7 


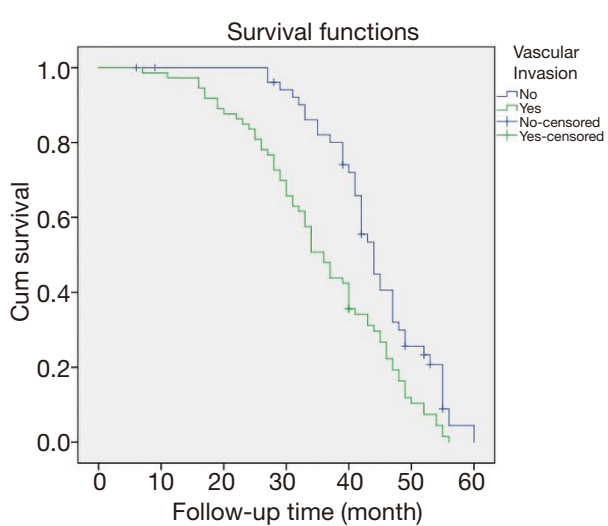

Figure 3 Correlation between vascular invasion and OS of postoperative cervical adenocarcinoma patients. OS, overall survival.

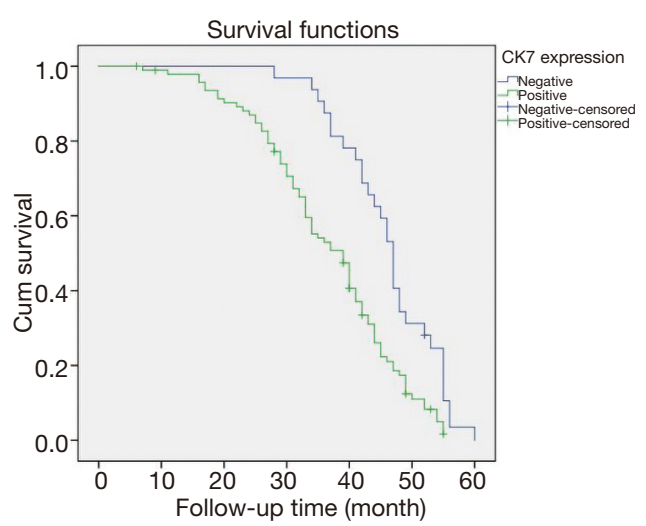

Figure 4 Correlation between CK7 expression and OS of postoperative cervical adenocarcinoma patients. CK7, cytokeratin 7; OS, overall survival.

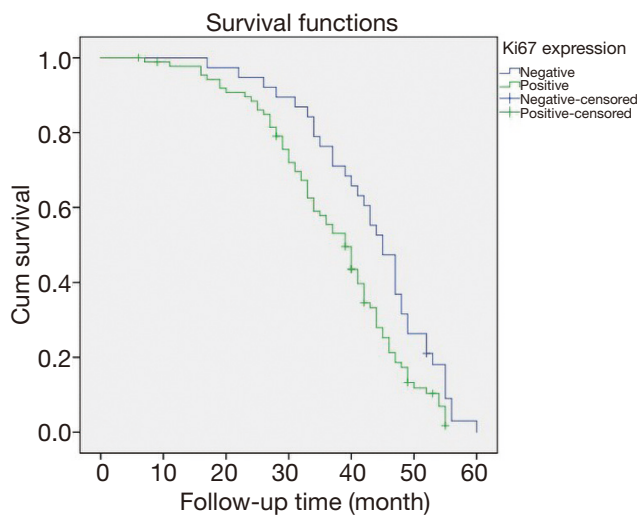

Figure 5 Correlation between Ki67 expression and OS of postoperative cervical adenocarcinoma patients. OS, overall survival. expression $(\mathrm{P}<0.001)$ (Figure 4). This study also found that the median survival time of patients with negative Ki67 expression was $43.36(40.10-46.62)$ months, which was higher than the $37.42(35.03-39.80)$ months of those with positive Ki67 expression, and the expression of Ki67 was correlated with patient survival $(\mathrm{P}<0.001)$ (Figure 5).

\section{Discussion}

Cervical cancer is a common malignant tumor in Chinese women. In recent years, the incidence of cervical squamous cell cancer has decreased due to the extensive screening of cervical cancer and precancerous lesions, which especially apply to cervical squamous cell cancer. However, the incidence of cervical adenocarcinoma has increased, accounting for about $10-20 \%$ of new cervical cancer cases every year; in particular, the incidence of patients aged under 35 years has increased significantly, and the prognosis is poor (17). Under the premise of no obvious clinical symptoms at early stage and high recurrence rate, it is of great clinical significance to identify molecular markers with strong specificity and high sensitivity, which can aid the diagnosis of cancer as early as possible and improve the prognosis and survival rate of cervical adenocarcinoma patients.

The marker CK7 is an alkaline cytokeratin with a molecular weight of $54 \mathrm{kDa}$. It is expressed in different parts of adenocarcinoma, and its expression pattern can be used to identify the primary site of metastasis adenocarcinoma. Studies have shown that CK7 is a positive promoter in the progression of malignant tumors, and its expression in malignant tumor tissue is higher than that in normal tissues $(18,19)$. The change of its expression concentration can change the synthesis of cytoskeleton components or the ability of cell deformation, which increases the risk of breaking through the basement membrane during the early development of cancer cells, leading to the change of the depth of infiltration of cancer cells into the periodic tissue. A study (20) showed that CK7 can induce the formation of local pseudopodia of cancer cells and improve the proliferation rate of cancer cells in the process of cell proliferation, indicating that the overexpression of $\mathrm{CK} 7$ may accelerate the metastasis of lymph nodes and cancer cells. Furthermore, the metastasis of cancer cells is also related to the degree of invasion and differentiation and tumor stage, which becomes the main reason for poor patient prognosis. The current study also found that patients with high degree of differentiation, $\geq 1 / 2$ myometrial invasion, 
vascular invasion, and lymph node metastasis also had high positive expression rate of CK7 protein. It was also found that the high expression of CK7 protein was associated with the degree of disease deterioration and poor prognosis of patients with cervical adenocarcinoma. The higher the positive rate of CK7 expression, the lower the OS.

The Ki67 protein is a kind of monoclonal antibody. It is composed of two polypeptide chains with molecular weight of 395 and $345 \mathrm{kD}$. It is encoded by a gene in band 5 , region 2 in the long arm of autosome 10 (10q25). It is a nuclear protein related to cell proliferation, and its function is considered to be closely related to cell mitosis. It is highly expressed when the cell is in the proliferative phase. There was expression of $\mathrm{Ki} 67$ in the nucleus at the G1, S, G2, and $M$ phases of cell cycle. but it was not expressed in G0 (cell resting state). In interphase, Ki67 is located in the dense fibrous component of nucleolus (21). The expression of Ki67 is associated with tumor proliferation, invasion, metastatic potential, and prognosis. Overexpression of Ki67 has a positive regulatory effect on cell cycle and accelerates the tumor occurrence and development (22). Research has shown that the expression of Ki67 in some tumors is closely related to the degree of malignancy and pathological classification of tumors, and its high expression is related to poor prognosis of malignant tumors (23). This study showed that the positive rate of Ki67 protein expression was higher in participants with more than $\geq 1 / 2$ myometrial invasion and higher tumor, node, metastasis (TNM) stage. Further study also found that a high level of Ki67 protein was associated with the degree of disease deterioration and poor prognosis of cervical adenocarcinoma patients. The higher the positive rate of Ki67 expression, the lower the OS.

\section{Conclusions}

In conclusion, CK7 and Ki67 proteins are highly expressed in patients with a higher malignant degree of cervical adenocarcinoma after operation. The CK7 and Ki67 proteins may be important regulatory factors in the development of cervical adenocarcinoma patients after operation, and the overexpression of CK7 and Ki67 may lead to worse prognosis. Therefore, CK7 and Ki67 may play an important role in cervical adenocarcinoma and may be a prognostic predictor of cervical adenocarcinoma patients.

\section{Acknowledgments}

Funding: None.

\section{Footnote}

Reporting Checklist: The authors have completed the REMARK reporting checklist. Available at https://dx.doi. org/10.21037/apm-21-1974

Data Sharing Statement: Available at https://dx.doi. org/10.21037/apm-21-1974

Conflicts of Interest: All authors have completed the ICMJE uniform disclosure form (available at https://dx.doi. org/10.21037/apm-21-1974). The authors have no conflicts of interest to declare.

Etbical Statement: The authors are accountable for all aspects of the work in ensuring that questions related to the accuracy or integrity of any part of the work are appropriately investigated and resolved. All procedures performed in this study involving human participants were in accordance with the Declaration of Helsinki (as revised in 2013). The study was approved by Jiangsu Cancer Hospital (No.: 20110584). All participants and their families were aware of the study, and all participants provided written informed consent before commencement of the study.

Open Access Statement: This is an Open Access article distributed in accordance with the Creative Commons Attribution-NonCommercial-NoDerivs 4.0 International License (CC BY-NC-ND 4.0), which permits the noncommercial replication and distribution of the article with the strict proviso that no changes or edits are made and the original work is properly cited (including links to both the formal publication through the relevant DOI and the license). See: https://creativecommons.org/licenses/by-nc-nd/4.0/.

\section{References}

1. Nakisige C, Trawin J, Mitchell-Foster S, et al. Integrated cervical cancer screening in Mayuge District Uganda (ASPIRE Mayuge): a pragmatic sequential cluster randomized trial protocol. BMC Public Health 2020;20:142.

2. Bray F, Ferlay J, Soerjomataram I, et al. Global cancer statistics 2018: GLOBOCAN estimates of incidence and mortality worldwide for 36 cancers in 185 countries. CA Cancer J Clin 2018;68:394-424. Erratum in: CA Cancer J Clin 2020;70:313.

3. Shi JF, Canfell K, Lew JB, et al. The burden of cervical 
cancer in China: synthesis of the evidence. Int J Cancer 2012;130:641-52.

4. Fujiwara H, Yokota H, Monk B, et al. Gynecologic Cancer InterGroup (GCIG) consensus review for cervical adenocarcinoma. Int J Gynecol Cancer 2014;24:S96-101.

5. Wang JD, Kong WM, Jiang H. FIGO 2018 staging of cervical cancer and related issues. Chinese Journal of Oncology 2020;42:94-8.

6. Liu P. Evaluation of clinical epidemiology of cervical cancer in mainland China in 13 years. Chinese Journal of Practical Gynecology and Obstetrics 2018;34:41-5.

7. Zhang X, Guo J, Cai Y, Sheng X. Whole-exome sequencing in cervical adenocarcinoma in mainland Chinese patients. Transl Cancer Res 2020;9:6889-99.

8. Li J, Sun XR, Zhao CQ, et al. Challenges in cervical adenocarcinoma screening. Chinese Journal of Clinical Obstetrics and Gynecology 2020;21:212-5.

9. Jenkins D, Molijn A, Kazem S, et al. Molecular and pathological basis of HPV-negative cervical adenocarcinoma seen in a global study. Int $\mathrm{J}$ Cancer 2020;147:2526-36.

10. Morris E, Roett MA. Genital cancers in women: cervical cancer. FP Essent 2015;438:18-23.

11. Serrano-Olvera A, Cetina L, Coronel J, et al. Emerging drugs for the treatment of cervical cancer. Expert Opin Emerg Drugs 2015;20:165-82 .

12. Wu NY, Zhang X, Chu T, et al. High methylation of ZNF582 in cervical adenocarcinoma affects radiosensitivity and prognosis. Ann Transl Med 2019;7:328.

13. Chhieng DC, Cangiarella JF, Zakowski MF, et al. Use of thyroid transcription factor $1, \mathrm{PE}-10$, and cytokeratins 7 and 20 in discriminating between primary lung carcinomas and metastatic lesions in fine-needle aspiration biopsy specimens. Cancer 2001;93:330-6.

14. Zheng H, Duan CX, Fu SL, et al. CK7 and P63 in NSCLC diagnosis and diagnostic value of combination. The Journal of Practical Medicine 2013;29:3524-7. 15. Niotis A, Tsiambas E, Fotiades PP, et al. ki-67 and

Cite this article as: $\mathrm{Wu} \mathrm{Y,} \mathrm{Lv} \mathrm{M,} \mathrm{Qian} \mathrm{T,} \mathrm{Shen} \mathrm{Y.} \mathrm{Correlation}$ analysis of Ki67 and CK7 expression with clinical characteristics and prognosis of postoperative cervical adenocarcinoma patients. Ann Palliat Med 2021;10(9):9544-9552. doi: 10.21037/ apm-21-1974
Topoisomerase IIa proliferation markers in colon adenocarcinoma. J BUON 2018;23:24-7.

16. Zhou Q, Wu XH, Liu JH, et al. Guidelines for the diagnosis and treatment of cervical cancer (fourth edition). Chinese Journal of Practical Gynecology and Obstetrics 2018;34:613-22.

17. Castellsagué X, Díaz M, de Sanjosé S, et al. Worldwide human papillomavirus etiology of cervical adenocarcinoma and its cofactors: implications for screening and prevention. J Natl Cancer Inst 2006;98:303-15.

18. Yun SP, Seo HI. Prognostic impact of immunohistochemical expression of CK7 and CK20 in curatively resected ampulla of Vater cancer. BMC Gastroenterol 2015;15:165.

19. Lyn J, Wang Y, Wang F, et al. Diagnostic value of SATB2, CK7 and CK20 in colorectal cancer. Zhonghua Bing Li Xue Za Zhi 2015;44:578-81.

20. Xu LP, Sun CY, Wang XY, et al. Detecting the role of the expression of three transcriptional regulation factors in identifying the pathological types of non-small cell lung cancer. Chinese Journal of Surgery of Integrated Traditional and Western Medicine 2015;21:227-30.

21. Shokouh TZ, Ezatollah A, Barand P. Interrelationships between Ki67, HER2/neu, p53, ER, and PR status and their associations with tumor grade and lymph node involvement in breast carcinoma subtypes: retrospectiveobservational analytical study. Medicine (Baltimore) 2015;94:e1359.

22. Hui EP, Ma BBY, Chan KCA, et al. Examination of ERCC1 status in circulating tumor cells as a prognostic tool for patients with nasopharyngeal carcinoma. Cancer Res 2017;77:abstr 3776.

23. Li WF. The expression level of Ki-67, Bcl-2, Bax and Caspase- 3 in brain glioma and its clinical significance. Shaanxi Medical Journal 2018;47:1377-9.

(English Language Editor: J. Jones) 